\title{
Understanding Anterior Knee Pain: Patellofemoral Pain Syndrome
}

Craig Nuttall

Brigham Young University - Provo

Blaine A. Winters

Brigham Young University - Provo, Blaine-Winters@byu.edu

Follow this and additional works at: https://scholarsarchive.byu.edu/facpub

Part of the Other Nursing Commons

\section{Original Publication Citation}

Nuttall, C. \& Winters, B. A. (2015). Understanding Anterior Knee Pain: Patellofemoral Pain Syndrome. Journal for Nurse Practitioners, (11)10. 1023-1035

\section{BYU ScholarsArchive Citation}

Nuttall, Craig and Winters, Blaine A., "Understanding Anterior Knee Pain: Patellofemoral Pain Syndrome" (2015). Faculty Publications. 5058.

https://scholarsarchive.byu.edu/facpub/5058 


\section{Understanding Anterior Knee Pain: Patellofemoral Pain Syndrome Craig Nuttall, MS, FNP-C, and Blaine A. Winters, DNP, ACNP-BC}
ABSTRACT
Patellofemoral pain syndrome (PFPS) is 1 of the most common causes of anterior knee pain and should be included in the differential diagnosis for anterior knee pain in every patient. PFPS is characterized as an overuse injury. The exact mechanism of injury is still under investigation, but it is believed to stem from maltracking of the patella. PFPS commonly presents in adults who complain of anterior knee pain with activity. This pain can lead to significant disability. When treated appropriately, disability and chronicity can be avoided or reduced.
Keywords: anterior knee pain, chondromalacia patella, knee pain, runner's knee, patellofemoral pain syndrome
(c) 2015 Elsevier, Inc. All rights reserved.

K nee pain is 1 of the most common reasons that a patient presents to the primary care setting. ${ }^{1,2}$ Of patients who present with knee pain, the most common location for that knee pain is in the anterior portion of the knee. ${ }^{3,4}$ When clinicians are considering the differential for knee pain, they should always consider patellofemoral pain syndrome (PFPS) in their differential diagnosis.

PFPS is the most common cause of knee pain seen in the outpatient setting. ${ }^{5}$ PFPS is characterized and categorized as an overuse injury. Although it is not a tendinopathy as most overuse injuries are, PFPS results from the repetitive motion of knee flexion. This overuse of the knee combined with several other factors can lead to irritation of the articular surface of the patella. This irritation leads to pain with bending the knee. PFPS, if left untreated, can lead to degeneration and long-lasting pain and disability. Eventually, permanent damage to the articular surface of the patella known as chondromalacia patella (CP) may develop.

PFPS is seen commonly in active adults who participate in activities such as running, basketball, and racket sports. ${ }^{7}$ Although it is 1 of the most commonly observed causes of knee pain in the outpatient setting, it is frequently misdiagnosed and improperly treated. Some of this may stem from the general lack of consistent terminology. Over the years, PFPS has been called jumper's knee, runner's knee, patellofemoral syndrome, CP, and idiopathic anterior knee pain.

Another cause of misdiagnosis and improper treatment is that the cause is often multifactorial and can be confusing. ${ }^{3,4}$ The purpose of this article is to clarify some of the confusion regarding PFPS and CP and to provide information on the current literature regarding the pathophysiology, diagnosis, and treatment of PFPS.

\section{THEORIES OF PATHOPHYSIOLOGY}

Debate as to the origin or cause of PFPS still occurs. Currently, several theories are thought to contribute to the development of PFPS. We will review the most widely accepted of these theories here.

\section{Maltracking of the Patella}

Most experts agree the mechanism of injury in PFPS is maltracking of the patella. ${ }^{3}$ In the normal knee, the patella should smoothly glide in between the 2 condyles of the femur in the trochlear groove. In individuals with PFPS, it has been observed that the patella does not track through the trochlear groove but instead takes an aberrant pathway during knee flexion. ${ }^{4}$ This pathway is usually over the lateral condyle of the femur. Abnormal tracking of the patella leads to increased pressure and grinding on the 
underside of the patella. Over time, it is thought that the increased friction and pressure can lead to softening and more severe damage of the cartilage on the underside of the patella, thus creating the more chronic and permanent condition called CP. ${ }^{6}$

\section{Muscle Weakness and Muscle Tension}

As stated previously, the theorized maltracking of the patella is usually in a lateral direction over the lateral condyle. The maltracking is almost never observed over the medial condyle because of the increased prominence. The cause of this maltracking remains elusive. There have been several theories regarding the cause of the maltracking. Most of these theories focus on muscle tension imbalance and muscle weakness. $^{4,8}$

Although many studies support that muscle weakness and/or muscle tension contribute to maltracking of the patella, there is little consensus on a single muscle group that is to blame. In reality, proper patellar tracking is a very dynamic motion that is influenced by several muscle groups including the quadriceps and gluteal muscles. ${ }^{4,8}$ As such, it is likely that maltracking of the patella can be caused by tightness or weakness in these muscle groups. ${ }^{\text {? }}$ Because of the complexity of this, it has been difficult to pin down a single cause of patellar maltracking.

\section{Quadriceps Dysfunction}

Several studies report that the quadriceps muscles play an important role in patellar tracking. ${ }^{10}$ Tightness in the vastus lateralis can lead to increased lateral pull on the patella and maltracking. ${ }^{10}$ Weakness in the vastus medialis can also lead to maltracking of the patella.

\section{Hip Rotation and Abduction Dysfunction}

The literature also identifies external hip rotator and hip abductor weakness as a cause of maltracking of the patella. ${ }^{9}$ It is hypothesized that weakness in the gluteal muscles leads to improper kinesiology that influences the patellar tracking.

\section{Inactivity and Increased Intensity of Physical Activity}

Imbalances in muscle strength and flexibility are seen in individuals who have been sedentary and have some degree of muscle atrophy because of inactivity. It is also seen in active individuals who have recently increased intensity of activity or have been involved in excessive activity. ${ }^{3}$

The result of maltracking of the patella leads to inflammation and irritation of the underside of the patella. The underside of the patella does not contain nociceptive nerve fibers so it is not the cause of the pain initially. It is hypothesized that the increased friction resulting from maltracking causes surrounding tissue inflammation and irritation leading to anterior knee pain.

\section{CLINICAL PRESENTATION}

\section{History}

The defining characteristics of PFPS include knee pain, typically in an anterior distribution without radiation, as well as pain with flexion of the knee with walking, running, or jumping. ${ }^{2}$ In more advanced stages of PFPS, the patient may have pain after sitting with the knee in the flexed position for an extended period of time. ${ }^{2}$ Typically, this is seen in those individuals who are very active or those who have recently intensified their physical activity too quickly. ${ }^{3}$ The pain associated with PFPS can lead to avoidance of the aggravating activities. The patient may also complain of knee swelling. ${ }^{3}$

\section{Physical Examination}

Physical examination of the patient with anterior knee pain should be comprehensive. The diagnosis of PFPS is one of exclusion. Other knee conditions that should be ruled out before a diagnosis of PFPS include meniscal tear, ACL and PCL tears, and other ligamentous damage. Knee evaluation should include inspection of the knee and surrounding tissues, palpation of the knee joint margins and patella, and active and passive range of motion of the knee. Special tests including tests to evaluate the ACL, PCL, and meniscus should be included in the examination.

There are several physical assessment modalities that are commonly used in the assessment of anterior knee pain and can be helpful in the identification of PFPS. It is important to note that many of these tests have not yet been rigorously tested. ${ }^{11}$ Although evidence is still lacking, some evidence suggests that using multiple tests to evaluate PFPS is effective in making a clinical diagnosis. Some of the more common tests used in the assessment of PFPS will be reviewed here. 


\section{Patellar Compression Test and Patellar Tilt Test}

The patellar compression test is performed while the patient is in the supine position. Have the patient extend both legs. While the legs are extended, apply direct pressure to the patella in a downward motion. After pressure is applied, the examiner should then tilt the patella from the inferior pole to apply pressure to the superior pole of the patella. A patient without PFPS should not complain of pain with this procedure. If the patient complains of discomfort or pain with this procedure, it is indicative of PFPS.

Although there is some evidence that this test is helpful in the diagnosis of PFPS, strong high-quality evidence is still lacking. ${ }^{11}$

\section{Patellar Apprehension Test or Active Instability Test}

The patellar apprehension test is performed by having the patient flex the knee to 30 degrees while the examiner tries to displace the patella. Watch the patients face for apprehension while performing this maneuver. Although this test is helpful in the diagnosis of PFPS, it should not be used as a stand-alone test in the diagnosis of PFPS. ${ }^{11}$

\section{Functional Knee Testing}

Functional testing of the knee is also important. Instruct the patient to perform the activities that cause the pain. This will help you evaluate the dynamics of the movement and determine the source of the pain. The most common aggravating activities are squatting down and walking up stairs. When there is pain associated with these activities, especially sitting for prolonged periods, walking stairs, or squatting, it is suggestive of PFPS. ${ }^{11}$

\section{DIAGNOSTIC TESTING}

The diagnosis of PFPS is made clinically and typically is a diagnosis of exclusion and does not require further imaging or testing. ${ }^{1}$ Radiology should be considered in patients with a history of trauma, surgery, or age greater than 50 years. ${ }^{1}$ Also, if the patient's pain is significant and is not improving after a trial of conservative treatment, radiography or magnetic resonance imaging (MRI) may be considered to evaluate for other possible pathologies. ${ }^{1}$

PFPS is primarily a clinical diagnosis. Therefore, radiographic imaging is usually not required. If trauma is involved or the history suggests the possibility of stress fractures, radiographic imaging can be considered.

Although MRI is effective in evaluation, advanced damage to the articular cartilage of the patella is not useful in the diagnosis of PFPS. ${ }^{12}$ MRI should be considered in patients who have atypical pain patterns for PFPS or who have instability or other findings on physical examination that are not consistent with PFPS. It should also be considered in patients with long-standing anterior knee pain. Several people with more chronic knee pain suffer from CP. Stage II and III CP can be accurately detected on MRI. ${ }^{12}$

\section{TREATMENT}

Treatment of PFPS should focus on correcting the cause of maltracking of the patella. Several treatment modalities have proven to be helpful.

\section{Physical Therapy}

Physical therapy may not be appropriate for all patients. However, those with significant pain or disability from PFPS may have better outcomes with physical therapy. Physical therapy has proven to be superior to home exercises. ${ }^{1}$ Physical therapy should focus on correcting the maltracking dysfunction by increasing the strength of the quadriceps muscles and hip rotation muscles as well as focusing on flexibility.

\section{Strengthening Exercises}

As previously mentioned, the quadriceps muscle plays a pivotal role in proper tracking of the patella in the trochlear grove. Any imbalance in the strength or flexibility of this muscle group will lead to increased risk for maltracking of the patella. The most commonly seen imbalance in the quadriceps muscles is weakness in the vastus medialas. Exercises should focus on strengthening of the vastus medialas and stretching of the vastus lateralis. Strengthening of all 4 quadriceps muscles has been proven to decrease pain and improve function in patients with PFPS. ${ }^{2,5,13}$ Strengthening exercises for the hip have also been proven to reduce pain and increase functionality in PFPS. ${ }^{5}$ It is recommended that strengthening exercises should always be included in the treatment of PFPS and that a combination of hip and quadriceps exercises is the most effective in treating PFPS. ${ }^{2,5,14}$ 


\section{Taping}

Some low-quality studies have shown that taping the knee in PFPS can be beneficial in reducing pain and increasing activity tolerance in PFPS. ${ }^{14}$ Several modalities of taping procedures have been proposed in the treatment of PFPS. Most use a non-elastictype tape to correct maltracking. Although taping alone has little effect on outcomes, it is useful during rehabilitation to reduce pain and increase the patient's ability to resume activity.

\section{Knee Bracing}

Knee bracing is widely used as treatment for anterior knee pain and PFPS. Knee braces have not been proven effective in correcting maltracking or improving outcomes in patients with PFPS. ${ }^{1,3}$ Knee braces should not be used routinely for PFPS.

\section{Medications}

Medications are not widely used for the treatment of PFPS. Nonsteroidal anti-inflammatory drugs may be used for short-term pain relief. There is no evidence to suggest that they influence the outcomes of PFPS significantly. ${ }^{1,14}$ Botulinum toxin type A injection into the knee can help reduce pain and increase knee flexion torque when used in patients with PFPS. ${ }^{15}$

\section{Surgical Treatment}

When conservative treatment has been ineffective in improving pain and functionality, surgical repair may then be considered. ${ }^{1}$ There are several procedures that are incorporated in the treatment of this disease. All procedures focus on cleaning up the damaged cartilage on the articular surface of the patella. Evidence shows that surgery should be considered as a last resort after failure of a comprehensive rehabilitation program.

\section{CONCLUSION}

PFPS is 1 of the most common causes of anterior knee pain encountered in the primary care setting. It should always be considered in the differential for anterior knee pain. Although we do not fully understand the cause of PFPS, it is clear that maltracking of the patella plays a major role in the development of PFPS. Patients who have anterior knee pain with walking stairs, squatting, and sitting for prolonged periods are more likely to have PFPS. ${ }^{11}$ Every patient diagnosed with PFPS should be given quadriceps and hip strengthening and stretching exercises. These have been proven to improve outcomes when used together in the treatment of PFPS. ${ }^{5}$ Patellar taping may be considered in some populations for pain reduction during rehabilitation. However, taping does not improve outcomes. Early diagnosis and treatment may lead to improved patient outcomes and decreased long-term disability related to PFPS. INP

\section{References}

1. Dixit S, DiFiori JP, Burton M, Mines B. Management of patellofemoral pain syndrome. Am Fam Physician. 2007;75(2):194-202.

2. Rodriguez-Merchan EC. Evidence based conservative management of patellofemoral syndrome. Arch Bone Jt Surg. 2014;2(1):4-6.

3. Petersen W, Ellermann A, Gösele-Koppenburg A, et al. Patellofemoral pain syndrome. Knee Surg Sports Traumatol Arthrosc. 2014;22(10):2264-2274.

4. Werner S. Anterior knee pain: an update of physical therapy. Knee Surg Sports Traumatol Arthrosc. 2014;22(10):2286-2294.

5. van der Heijden RA, Lankhorst NE, van Linschoten R, Bierma-Zeinstra SM, van Middelkoop M. Exercise for treating patellofemoral pain syndrome. An abridged version of Cochrane Systematic Review. Eur J Phys Rehabil Med. $2015 \mathrm{Jul} 9$ [Epub ahead of print].

6. Tuna BK, Semiz-Oysu A, Pekar B, Bukte Y, Hayirlioglu A. The association of patellofemoral joint morphology with chondromalacia patella: a quantitative MRI analysis. Clin Imaging. 2014;38(4):495-498.

7. Baquie $P$, Brukner P. Injuries presenting to an Australian sports medicine centre: a 12-month study. Clin J Sport Med. 1997;7(1):28-31.

8. Thomeé R, Augustsson J, Karlsson J. Patellofemoral pain syndrome: a review of current issues. Sports Medicine (Auckland, N.Z.). 1999;28(4):245-262.

9. Lankhorst NE, Bierma-Zeinstra SMA, van Middelkoop M. Factors associated with patellofemoral pain syndrome: a systematic review. Br J Sports Med. 2013;47(4):193-206

10. Santos TRT, Oliveira BA, Ocarino JM, Holt KG, Fonseca ST. Effectiveness of hip muscle strengthening in patellofemoral pain syndrome patients: a systematic review. Braz J Phys Ther. 2015;19(3):167-176.

11. Cook C, Mabry L, Reiman MP, Hegedus EJ. Best tests/clinical findings for screening and diagnosis of patellofemoral pain syndrome: a systematic review. Physiotherapy. 2012;98(2):93-100.

12. Mattila VM, Weckström M, Leppänen V, Kiuru M, Pihlajamäki H. Sensitivity of MRI for articular cartilage lesions of the patellae. Scand J Surg. 2012;101(1):56-61.

13. Bizzini M, Childs JD, Piva SR, Delitto A. Systematic review of the quality of randomized controlled trials for patellofemoral pain syndrome. J Orthop Sports Phys Ther. 2003:33(1):4-20.

14. Rixe JA, Glick JE, Brady J, Olympia RP. A review of the management of patellofemoral pain syndrome. Phys Sportsmed. 2013;41(3):19-28.

15. Chen JT-N, Tang AC-W, Lin S-C, Tang SF-T. Anterior knee pain caused by patellofemoral pain syndrome can be relieved by Botulinum toxin type A injection. Clin Neurol Neurosurg. 2015;129(suppl 1):S27-S29.

Craig Nuttall, MS, FNP-C, and Blaine A. Winters, DNP, ACNP-BC, are assistant teaching professors at the College of Nursing, Brigham Young University in Provo, UT. Craig Nuttall can be reached at craig-nuttall@byu.edu. In compliance with national ethical guidelines, the authors report no relationships with business or industry that would pose a conflict of interest.

$1555-4155 / 15 / \$$ see front matter

(c) 2015 Elsevier, Inc. All rights reserved.

http://dx.doi.org/10.1016/j.nurpra.2015.08.029 